\title{
Loneliness in Young Adulthood: a Protocol for a Scoping Review of the Quantitative and Qualitative Literature.
}

\section{Emma Kirwan ( $\nabla$ emma.kirwan@ul.ie )}

University of Limerick https://orcid.org/0000-0001-8536-023X

Páraic S O'Súilleabháin

University of Limerick

Annette Burns

Institute of Public Health in Ireland

Jennifer McMahon

University of Limerick

\section{Sarah Summerville}

University of Limerick

Ann-Marie Creaven

University of Limerick

\section{Protocol}

Keywords: Loneliness, young adulthood, mental health, emerging adulthood, scoping review

Posted Date: March 18th, 2021

DOl: https://doi.org/10.21203/rs.3.rs-316759/v1

License: (1) This work is licensed under a Creative Commons Attribution 4.0 International License. Read Full License 


\section{Abstract}

\section{Background}

Loneliness refers to the distressing feeling that accompanies the experience of perceiving the quantity or quality of one's social relationships as inadequate (1). There is increasing recognition of the prevalence of loneliness in young adults. Despite this, there is no existing scoping review on loneliness in young adulthood. Young adults (18-25 years) are in a critical life stage involving diverse social, demographic, biological and cognitive transitions which may affect the development of loneliness. Evidence that loneliness is a risk factor for poorer mental and physical health further emphasises the need to understand the experience in this age group. Therefore, the aim of our scoping review is to provide a summary of the quantitative and qualitative literature on loneliness in young adulthood.

\section{Methods}

The proposed scoping review will follow the Joanna Briggs Institute methodology and Arksey and O'Malley's (2005) framework for scoping reviews. Peer-reviewed journal articles and grey literature in the form of reports or difficult-to-locate studies will be identified by: (a) electronic database searching, (b) contacting national and international researchers in the field, and (c) by posting general requests for relevant information on Twitter. We will include all study designs published in English from 2000 to 2021 where loneliness (defined as subjective) is a key focus of the work and the mean age of participants is $\geq$ 18 and $\leq 25$ years. Editorials, commentaries, opinion pieces, dissertations, and book chapters will be excluded. Articles will be selected for inclusion following screening of titles/abstracts succeeded by fulltext screening. Results will be presented in a narrative form to provide a descriptive summary of the literature on loneliness in young adults.

\section{Discussion}

The results of this scoping review will provide an up-to-date overview of available research related to loneliness in young adults and will inform our future research in the area. Results will be shared through a peer-reviewed journal publication and conference presentations.

\section{Registration}

This protocol is registered with the Open Science Framework (OSF) available at: https://osf.io/jfcmp.

\section{Background}

Loneliness has been defined as the distressing feeling that accompanies the experience of perceiving one's social relationships as inadequate, either quantitatively or qualitatively (1). Although loneliness is often viewed as a problem of older adulthood, the need to belong and maintain social connections with others is observed across the lifespan and all age groups are susceptible to loneliness when these needs are not satisfied $(2,3)$. In fact, loneliness is particularly prevalent in young adults; about $40 \%$ of young 
adults (16-24 years) report feeling lonely often or very often (4). Despite its prevalence, loneliness has only recently been recognised as an important issue in young adult groups and to our knowledge, there is no existing scoping review on the topic.

Young adults are of particular interest in loneliness research considering that this life stage which marks the transition from adolescence to adulthood is characterised by important developmental milestones, cognitive and physical maturation, identity exploration, and increased autonomy which may place this age group at increased risk for loneliness (5-7). While short-term, or transient, loneliness is unlikely to have long-lasting consequences and might even function to motivate connection with others $(5,8,9)$, almost one in ten (9\%) young adults will experience persistent loneliness (10). Individuals who suffer from persistent, or chronic, loneliness tend to experience more long-term psychosocial problems and negative health outcomes, relative to those with short term loneliness (9).

The prevalence of loneliness in young adults is concerning considering that the experience is a wellestablished risk factor for poorer physical and mental health $(8,11)$. Lonely young adults are more likely to experience depressive symptoms, to have poorer sleep quality, poorer self-rated general health, and altered cortisol activity (12-15). Although the long-term health consequences of loneliness may not become apparent until later life, the negative experience and behaviours associated with loneliness may increase an individual's risk early in life. Young adults who experience loneliness are more likely to engage in health risk behaviours, to use negative stress coping strategies, and to have increased risk of disability and lower income in midlife $(16,17)$.

Recognising that loneliness may have negative implications for young adults' health emphasises the need to consider the factors associated with loneliness in this group. Young adults have a similar, if not higher, prevalence of loneliness compared to older adults $(10,18)$; however, research has tended to focus on the latter. Although loneliness is a universal experience at the individual level, the risk factors associated with loneliness and the influence of these factors can vary across the lifespan. For example, the association between loneliness and less contact with friends is strongest for young adults (19-34 years) compared to early (35-49 years) and late (50-65 years) middle-aged adults (19). Therefore, there is a need for a greater understanding of the specific risk factors for loneliness in young adulthood and the target groups for intervention, beyond those already identified (20).

Given its complex nature, it is unsurprising that research on loneliness spans many disciplines using different theoretical perspectives; some approaches are specific to loneliness, but broader theories have also been applied in this area. A leading approach is the cognitive discrepancy model (2), which emphasises the role of individual traits and cognitive processes in the subjective experience of loneliness. In contrast, the social needs approach (21) views loneliness as a multidimensional construct capturing an unmet need for intimate attachment (emotional loneliness) or meaningful friendships (social loneliness). Loneliness has attracted interest from sociologists, who have described loneliness as a product of wider society (22). Other approaches relevant in loneliness research relate to social connectedness, such as Bowlby's (23) attachment theory which highlights the influence of early 
attachments on future psychological well-being. Finally, broader developmental approaches, such as Bronfenbrenner's (24) model of ecological systems, have demonstrated usefulness in loneliness research (25). Theoretical approaches applied in loneliness research are not mutually exclusive and may overlap. Therefore, the research on loneliness in young adults is likely to be somewhat disparate; yet no review of loneliness in young adults has specifically considered the theoretical approaches employed in this area of research.

\section{Definition of loneliness and young adults}

Throughout this review, we will use the term loneliness to refer to the distressing feeling that accompanies the experience of perceiving one's social relationships as inadequate, either quantitatively or qualitatively (1). Across age groups, loneliness is subjective and is only weakly associated with objective measures of contact with friends and family $(18,26)$. Loneliness is distinct from social isolation, solitude, and living alone, such that these situations may not be unpleasant; adolescents' perception of loneliness in comparison to aloneness suggests that loneliness is seen as aversive and related to negative affect like sadness, whereas aloneness is not perceived negatively (27).

There is no defined threshold for transition from adolescence to adulthood. Due to social and economic changes in contemporary societies, the process of achieving adulthood may continue for several years until the mid-to-late twenties (28). Many of the traditional markers of adulthood, such as the age of first marriage, are now being achieved later in life than in previous years (29). This prolonged development from adolescence to adulthood has been termed "extended adolescence" by some developmental researchers (30). Others have described young, or "emerging", adulthood as a distinct period of the life course from age 18 to 25 years (6). Emerging adulthood is characterised by instability, possibilities, identity exploration, self-focus, and feeling in-between (7), and represents a separate life stage marking the transition from adolescence to adulthood involving a unique constellation of demographic, social, and psychological correlates (31), which may have bearing on the development of loneliness. Because the changes associated with the transition from adolescence to adulthood may be particularly relevant to the experience of loneliness, our review aims to focus on young or "emerging" adulthood.

\section{Review aims and objectives}

Despite increasing research interest in loneliness in young adults $(16,32,33)$, our preliminary search of the literature (Psyclnfo and Scopus on $9^{\text {th }}$ February 2021) revealed no existing scoping reviews on the topic. Given the broad nature of this topic, a scoping review, rather than a systematic review, will summarise the existing literature on loneliness in young adulthood. Scoping reviews are particularly useful for disparate literature or topics with emerging evidence (34). Like systematic reviews, scoping reviews use a methodologically rigorous process to find and synthesise literature (35). Rather than follow a highly focused research question, scoping reviews aim to identify all relevant literature in an area (36). Therefore, conducting a scoping review is an iterative process which enables us to make (and document) necessary changes to the search strategy to identify relevant studies. We hope to provide a foundation to 
inform subsequent loneliness research in young adults ensuring that studies are based on the most up-todate knowledge in the area.

The aim of our scoping review is to provide an up-to-date summary of the qualitative and quantitative literature on loneliness in young or "emerging" adulthood. Our main objectives are (a) to identify what theoretical approaches are used in research on loneliness in young adulthood, (b) to summarise the evidence examining the correlates of and risk factors for loneliness in young adulthood, (c) to summarise how loneliness is conceptualised and measured in research in young adults, (d) to summarise the evidence on sex-gender differences in loneliness in young adults, (e) to identify and summarise the young adult groups that have been included in previous loneliness literature; in doing so, we may identify groups of young adults that are currently underrepresented in this area of research.

\section{Methods}

\section{Study design}

We will conduct a scoping review to provide a descriptive summary of the available research on loneliness in young adults. Our review will aggregate and articulate the findings without formal analysis of the quality of the available studies.

This review is informed by the Joanna Briggs Institute (JBI) framework for scoping reviews (37) and Arksey and O'Malley's (36) seminal work in scoping review methodology. This protocol follows the key stages proposed by Arksey and O'Malley (36): (a) identify the research question, (b) search for relevant studies, (c) select studies, (d) chart the data, (e) collate, summarise and report the results.

\section{Identifying the research question}

Considering the aim and objectives of our scoping review, the following research question has been formulated: What is known from the available literature about loneliness in young adults? In addition, several sub-questions which will inform data charting and the reporting of results. These are:

1. What theoretical approaches have been used in research on loneliness in young adults?

2. What correlates of and risk factors for loneliness have been studied in research on young adults ?

3. In what ways has loneliness been conceptualised and measured in research on young adults?

4. Are sex-differences in loneliness observed?

5 . What young adult groups have been researched in previous loneliness literature?

\section{Information sources and search strategy}

\section{Electronic databases}

The following electronic databases will be searched: Scopus, PubMed, PsycArticles, Psyclnfo, Medline, ScienceDirect, and Applied Social Sciences Index and Abstracts (ASSIA). The search will be limited to 
papers published from 2000 to 2021 . This year limit is proposed for two reasons. First, Arnett's (6) seminal paper on emerging adulthood was published in the year 2000; considering that this is a highly cited paper, we expect to find more peer-reviewed research on our target population after this year. Second, this year limit is in line with our aim to provide an up-to-date summary of the research on loneliness in young adults.

The search strategy includes combinations of keywords and terms related to our population group of interest (young adults) and loneliness (Table 1). The search strategy will be adapted for each database listed above (example attached in Additional File 1). Following JBI guidance on scoping review methodology, a preliminary search of two databases (Psyclnfo and Medline) will be carried out. The search terms may be modified to ensure that the search strategy is comprehensive.

Table 1

Keywords and search terms to be used in the database searches.

\begin{tabular}{|ll|}
\hline Population & Issue \\
\hline Young* adult* & Lonel* \\
\hline Youth* & Subjective social isolation \\
\hline Young pe* & Perceived social isolation \\
\hline Emerging adult* & \\
\hline Early adult* & \\
\hline Adolescen* & \\
\hline Teenage* & \\
\hline
\end{tabular}

\section{Grey literature and manual searches}

The term 'grey literature' is commonly understood to refer to electronic and print sources (e.g., reports, working papers, dissertations) that are not controlled by commercial publishing organisations (38). Prior to devising a search strategy for grey literature, we considered the benefits and challenges of its inclusion in scoping reviews.

Overall, including grey literature provides the opportunity to produce a more comprehensive and timeliness picture of available evidence in an area. The emphasis on knowledge transfer in areas related to mental health, such as loneliness research, means that the publication of research in a scientific journal may not be the best way to ensure that findings are communicated to those most interested in the topic (e.g., youth mental health organisations, policy makers). It is likely that information relevant to loneliness in young adulthood will be found in reports by organisations or government bodies interested 
in youth mental health (e.g., Jigsaw, SpunOut.ie, National Youth Council of Ireland) and these sources may not be retrieved in database searching.

Of course, there are limitations to grey literature searching. First, the time consuming and heterogeneous nature of grey literature must be considered (39). Tricco (40) recounted the challenge in screening approximately 5000 titles and abstracts to include grey literature in a scoping review. Given that abstracts are not available for many grey literature documents, the full text of individual documents may need to be reviewed in order to determine eligibility. Second, there is no gold-standard method to systematically search for grey literature. Grey literature search strategies are better described as systematic rather than replicable; even if our search is replicable, other researchers may not retrieve the same results on replication. Previous review papers lack adequate descriptions of the search for and synthesis of results from grey literature sources (39). As such, we aim to be explicit in our methodology by outlining our considerations and including a level of detail in the final scoping review paper that maximises the transparency of our grey literature search.

A key aspect of our grey literature search is maximising search sensitivity while retrieving search results that are feasible for screening. In line with this, we considered the use of search engines such as Google to source grey literature. Although search engines are easily accessible, the low specificity and sensitivity of this method, as well as the likelihood of finding documents already found elsewhere, reduces its effectiveness. Google results are influenced by geographical location, previous search history, and popularity, meaning that the replicability and consistency of the search results may be compromised (41).

Having considered the importance of including grey literature, the advantages and disadvantages of different strategies, and the current variability in approaches to grey literature, we have devised a complementary search strategy which specifically relates to sourcing grey literature documents in the form of reports or difficult to locate studies relating to loneliness in young adults. We have chosen two methods to source grey literature in our scoping review: (1) by contacting national and international researchers in the field via I-LINK (International Loneliness and Isolation NetworK), and (2) by posting general requests for relevant information on Twitter and by mentioning relevant organisations ('@organisation') in such tweets, as described in Adams et al. (38).

We will identify key experts in the area via I-LINK; these researchers will be contacted and asked to nominate documents for inclusion in the review. By contacting other researchers in the area we may also identify studies in progress or recently published studies that are not retrieved in database searches. We will also post general requests targeting national and international organisations on the social media site Twitter and ask that other users repost these tweets, so as to increase the potential viewers. A previous review found this to be a more efficient process than email or professional press requests (38). We will receive and consider documents for inclusion in the review up until the point of submission to a journal. The reference lists of previous reviews and articles eligible for inclusion (from either the core search or the complementary grey literature search) will be examined to locate additional relevant studies.

\section{Identifying relevant studies}


Conducting a scoping review is an iterative process $(34,37)$, meaning that some changes to the inclusion and exclusion criteria may be necessary following trial study selection. Any deviations from this protocol will be clearly detailed in the 'Methods' section of the final scoping review paper.

\section{Inclusion criteria}

a. Research where loneliness, defined as 'subjective' or 'perceived social isolation', is a key focus of the work (determined by the inclusion of a relevant aim, objective, or research question related to understanding loneliness) or if the study measures and reports findings related to loneliness under a broader concept, such as 'psychological well-being', 'mental health', or similar.

b. Qualitative, quantitative, mixed-methods, systematic reviews, meta-analyses, meta-syntheses articles.

c. Mean age of participants is $\geq 18$ and $\leq 25$ years. Emerging adults share some characteristics with the life stages of adolescence and adulthood; samples that overlap our operationalization of emerging adulthood (e., $\geq 18$ and $\leq 25$ years) with a wider age range, but that report a mean age $\geq$ 18 and $\leq 25$ years will be included.

d. Studies which focus on loneliness in clinical or specific subpopulations, such as young adults with Type 1 diabetes (42), will be eligible for inclusion but may be summarised separately to studies of general or community samples in the results section of the review.

e. Research articles are not limited by setting or geographical location.

f. Articles published in English (that is the only language of the researchers).

g. Publications in the form of peer-reviewed articles or grey literature to include reports from relevant NGOs (e.g., SpunOut.ie, Jigsaw), loneliness organisations (e.g., Campaign to End Loneliness) and government bodies (e.g., National Youth Council of Ireland) or difficult to locate studies (i.e., empirical research which has only recently or not yet been published in a journal).

\section{Exclusion criteria}

a. Articles not related to loneliness (such as those only focused on social isolation or living alone) or articles where loneliness is not considered a key aspect (e.g., loneliness is only included as a control variable).

b. Articles where the mean age of participants is $<18$ or $>25$ years, where the age of the population is not clearly described, or studies with a wider age range where data for emerging adults (i.e., $\geq 18$ and $\leq 25$ years) cannot be extrapolated.

c. Editorials, commentaries, opinion pieces, dissertations, and book chapters.

d. Articles published in a language other than English.

\section{Study selection}


Articles retrieved from the database searches will be added to EndNoteX9 (Clarivate Analytics) to manage the references. The first author (EK) will conduct database searching and removal of duplicates. Articles will be screened in two phases. First, the titles and abstracts of all articles retrieved from the database search, as well as reports and empirical studies located from additional sources, will be screened for eligibility by EK. Fifty percent of the titles and abstracts will be independently screened by a second reviewer (SS). Studies and reports that do not meet our initial inclusion criteria will be excluded. Second, the full-text of the remaining articles that appear to fulfil the inclusion criteria will be obtained and examined for eligibility by EK. Fifty percent of the full-texts will be independently reviewed by SS. Studies that do not meet the inclusion criteria at this stage will be excluded and the reason for exclusion noted. Any disagreement between reviewers in decisions regarding the eligibility of studies will be resolved by a third reviewer. Rayyan QCRI software will be used to manage study selection. The study selection process, including reasons for exclusion, will be presented in a PRISMA flow diagram in the final paper.

\section{Data charting}

Data charting will be carried out for all papers by one reviewer (EK). A proportion of the eligible papers (minimum of $10 \%$ ) will also be assessed by SS for accuracy. The data charting form will be pre-piloted on a random selection of articles to ensure all relevant information is extracted. Uncertainty regarding the charting of results will be resolved through discussion, including a third author if necessary. The charting table will include the following details:

Bibliographic information.
a. Author(s) information.
b. Year of publication.
c. Journal.
d. Article title.

Study information.

a. Country of origin.

b. Study setting (e.g., community setting, online survey, university).

c. Quantitative, qualitative, mixed-methods, review.

d. Study design.

e. Sample size.

f. Age range and average age of sample.

g. Sample characteristics (i.e., ethnic groups, nationality, university or non-university sample).

h. Gender distribution.

Subject matter information. 
a. Study aims and objectives.

b. If the study specifically concerns loneliness during the Covid-19 pandemic.

c. Theoretical approach used: whether specific to loneliness (e.g., the cognitive discrepancy model (2), social needs approach (21)), or a broader paradigm (e.g., ecological systems theory (24)).

d. Typology of loneliness: Ioneliness as unidimensional or multidimensional (e.g., Weiss (21) subtypes of social and emotional loneliness).

e. Assessment or measurement of loneliness: which psychometric measures of loneliness were used? Were indirect or direct measures used, or both? Were psychometric measures adapted (i.e., was the wording of the psychometric measure adapted, was the assessment adapted to make it suitable for an online survey?).

f. Sex-gender differences (if assessed in quantitative studies): are sex-gender differences reported for overall loneliness or for subtypes? What is the $p$ value and effect size?

g. Correlates and risk factors assessed (only in quantitative studies): what is the $p$ value and strength of the association?

h. Qualitative research approach: e.g., grounded theory, IPA (only in qualitative studies).

i. Key themes (only in qualitative studies).

j. Main findings and conclusions.

\section{Summarising of results}

The reporting of results will be guided by the Preferred Reporting Items for Systematic reviews and MetaAnalysis extension for Scoping Reviews (PRISMA-ScR) (43). A completed PRISMA-P checklist is included as an additional file to this protocol (Additional File 2). The aim of our scoping review is to provide a descriptive summary of the available evidence; the quality of the studies included will not be assessed. All findings will be included in a narrative review. Studies which report on clinical or specific subpopulations, such as individuals with inflammatory bowel disease (44) or young adults with Type 1 diabetes (42), may be summarised separately to studies which focus on community samples. The reporting of our results will be guided by our research questions. The theoretical approaches used in research on loneliness in young adults (RQ1), correlates of and risk factors for loneliness (RQ2), and the means of conceptualising and measuring loneliness (RQ3) will be recorded in tables with accompanying narrative summaries. We will identify commonalities across studies and collate the extracted data. Observed sex differences (RQ4) and the young adult groups previously researched (RQ5) will be reported using a narrative summary. Included grey literature reports will also be summarised in the narrative review. The findings from both kinds of literature will be combined to address our primary aim in providing a summary of the quantitative and qualitative literature on loneliness in young adults.

\section{Discussion}


This review aims to be the first scoping review to provide a descriptive summary of the available literature on loneliness in young, or "emerging", adults. Specifically, we aim to summarise: (a) the theoretical approaches used in this area, (b) the risk factors associated with loneliness in this group, (c) the conceptualisation and means of measuring loneliness in young adults, (d) the observed sex differences, if any, in loneliness in young adults, and (e) the young adult groups that have been included in previous loneliness research.

Following the completion of our scoping review, results will be shared through a peer-reviewed publication and conference presentations. This review forms part of a larger project which aims to identify the risk factors for loneliness in young adulthood. The results of this scoping review will provide an up-to-date overview of available research related to loneliness in young adults and will inform our future research in the area.

\section{Limitations}

The potential limitations to our scoping review include that older studies (published before the year 2000) and documents that are not available in electronic databases or online may be missed. However, we anticipate that the majority of relevant literature will be captured by our search strategies, including grey literature.

\section{Abbreviations}

JBI: Joanna Briggs Institute; ASSIA: Applied Social Sciences Index and Abstracts; I-LINK: International Loneliness and Isolation Network; NGO: non-governmental organisation; EK: Emma Kirwan; SS: Sarah Summerville; PRISMA: Preferred Reporting Items for Systematic Reviews and Meta-analyses; IPA: interpretative phenomenological analysis; PRISMA-ScR: Preferred Reporting Items for Systematic reviews and Meta-Analysis extension for Scoping Reviews; PRISMA-P: Preferred Reporting Items for Systematic reviews and Meta-Analysis Protocols; RQ: research question.

\section{Declarations}

\section{Ethics approval and consent to participate}

Not applicable.

\section{Consent for publication}

Not applicable.

\section{Availability of data and materials}

Not applicable. 


\section{Competing interests}

The authors declare that they have no competing interests.

\section{Funding}

The study is supported by the Department of Psychology, University of Limerick. The funder did not play any role in the development of this protocol.

\section{Authors' contributions}

EK is the guarantor of the study. EK, $P S O, A B$, and $A M C$ were involved in the conception and design of the study protocol. EK, PSO, AB, JMM, and AMC contributed to the drafting and revising of the protocol manuscript. SS was involved in the planning of study selection and data charting. The final manuscript was approved by all authors.

\section{Acknowledgements}

Not applicable.

\section{References}

1. Perlman D, Peplau LA. Toward a social psychology of loneliness. Personal Relationships. 1981;3:3156.

2. Perlman D, Peplau LA. Theoretical approaches to loneliness. Loneliness: A sourcebook of current theory, research and therapy. New York: John Wiley and Sons; 1982. p. 123-34.

3. Baumeister RF, Leary MR. The need to belong: desire for interpersonal attachments as a fundamental human motivation. Psychological Bulletin. 1995;117(3):497.

4. BBC Loneliness Experiment. Who feels lonely? The results of the world's largest loneliness study [Internet]. 2018 [cited 24 Feb 2021]. Available from: https://www.bbc.co.uk/programmes/articles/2yzhfv4DvqVp5nZyxBD8G23/who-feels-lonely-theresults-of-the-world-s-largest-loneliness-study.

5. Qualter P, Vanhalst J, Harris R, Van Roekel E, Lodder G, Bangee M, et al. Loneliness across the life span. Perspectives on Psychological Science. 2015;10(2):250-64.

6. Arnett JJ. Emerging adulthood: A theory of development from the late teens through the twenties. American Psychologist. 2000;55(5):469.

7. Arnett JJ, Mitra D. Are the features of emerging adulthood developmentally distinctive? A comparison of ages 18-60 in the United States. Emerging Adulthood. 2018;8(5):412-9.

8. Hawkley LC, Cacioppo JT. Loneliness matters: A theoretical and empirical review of consequences and mechanisms. Annals of Behavioral Medicine. 2010;40(2):218-27. 
9. Hawkley LC, Capitanio JP. Perceived social isolation, evolutionary fitness and health outcomes: a lifespan approach. Philosophical Transactions of the Royal Society B: Biological Sciences. 2015;370(1669):20140114.

10. Victor CR, Yang K. The prevalence of loneliness among adults: a case study of the United Kingdom. The Journal of Psychology. 2012;146(1-2):85-104.

11. Cacioppo JT, Hawkley LC, Thisted RA. Perceived social isolation makes me sad: 5-year cross-lagged analyses of loneliness and depressive symptomatology in the Chicago Health, Aging, and Social Relations Study. Psychology and Aging. 2010;25(2):453.

12. Doane LD, Adam EK. Loneliness and cortisol: Momentary, day-to-day, and trait associations. Psychoneuroendocrinology. 2010;35(3):430-41.

13. Goosby BJ, Bellatorre A, Walsemann KM, Cheadle JE. Adolescent loneliness and health in early adulthood. Sociological Inquiry. 2013;83(4):505-36.

14. Vanhalst J, Goossens L, Luyckx K, Scholte RH, Engels RC. The development of loneliness from midto late adolescence: Trajectory classes, personality traits, and psychosocial functioning. Journal of Adolescence. 2013;36(6):1305-12.

15. Pressman SD, Cohen S, Miller GE, Barkin A, Rabin BS, Treanor JJ. Loneliness, social network size, and immune response to influenza vaccination in college freshmen. Health Psychology. 2005;24(3):297.

16. Von Soest T, Luhmann M, Gerstorf D. The development of loneliness through adolescence and young adulthood: Its nature, correlates, and midlife outcomes. Developmental Psychology. 2020.

17. Matthews T, Danese A, Caspi A, Fisher HL, Goldman-Mellor S, Kepa A, et al. Lonely young adults in modern Britain: Findings from an epidemiological cohort study. Psychological Medicine. 2019;49(2):268-77.

18. Luhmann M, Hawkley LC. Age differences in loneliness from late adolescence to oldest old age. Developmental Psychology. 2016;52(6):943-59.

19. Franssen T, Stijnen M, Hamers F, Schneider F. Age differences in demographic, social and healthrelated factors associated with loneliness across the adult life span (19-65 years): a cross-sectional study in the Netherlands. BMC Public Health. 2020;20(1):1-12.

20. Fried L, Prohaska T, Burholt V, Burns A, Golden J, Hawkley L, et al. A unified approach to loneliness. 2020.

21. Weiss RS. Loneliness: The experience of emotional and social isolation. Cambridge: MIT Press; 1973.

22. Bauman Z. Liquid modernity. Cambridge: Polity; 2000.

23. Bowlby J. Attachment and Loss, Vol. 1. Attachment. New York: Basic Books; 1969.

24. Bronfenbrenner U. Ecological systems theory. Annals of Child Development. 1989;6:187-249.

25. Chipuer HM. Dyadic attachments and community connectedness: Links with youths' loneliness experiences. Journal of Community Psychology. 2001;29(4):429-46. 
26. Rokach A. Loneliness updated: An introduction. The Journal of Psychology. 2012;146(1-2):1-6.

27. Buchholz ES, Catton R. Adolescents' perceptions of aloneness and loneliness. Adolescence. 1999;34(133):203-4.

28. Reifman A, Arnett JJ, Colwell MJ. Emerging adulthood: Theory, assessment and application. Journal of Youth Development. 2007;2(1):37-48.

29. Arnett JJ. Adolescence and Emerging Adulthood: A Cultural Approach 2nd Edition: Prentice Hall; 2004.

30. Steinberg L. Age of opportunity: Lessons from the new science of adolescence: Houghton Mifflin Harcourt; 2014.

31. Wood D, Crapnell T, Lau L, Bennett A, Lotstein D, Ferris M, et al. Emerging adulthood as a critical stage in the life course. Handbook of life course health development: Springer, Cham; 2018. p. 12343.

32. Beam CR, Kim AJ. Psychological sequelae of social isolation and loneliness might be a larger problem in young adults than older adults. Psychological Trauma: Theory, Research, Practice, and Policy. 2020;12(S1):S58.

33. Fried L, Prohaska T, Burholt V, Burns A, Golden J, Hawkley L, et al. A unified approach to loneliness. The Lancet. 2020;395(10218):114.

34. Levac D, Colquhoun H, O'Brien KK. Scoping studies: advancing the methodology. Implementation Science. 2010;5(1):69.

35. Pham MT, Rajić A, Greig JD, Sargeant JM, Papadopoulos A, McEwen SA. A scoping review of scoping reviews: advancing the approach and enhancing the consistency. Research Synthesis Methods. 2014;5(4):371-85.

36. Arksey H, O'Malley L. Scoping studies: towards a methodological framework. International Journal of Social Research Methodology. 2005;8(1):19-32.

37. Peters MD, Godfrey CM, Khalil H, McInerney P, Parker D, Soares CB. Guidance for conducting systematic scoping reviews. International Journal of Evidence-Based Healthcare. 2015;13(3):141-6.

38. Adams J, Hillier-Brown FC, Moore HJ, Lake AA, Araujo-Soares V, White M, et al. Searching and synthesising 'grey literature'and 'grey information'in public health: critical reflections on three case studies. Systematic Reviews. 2016;5(1):1-11.

39. Mahood Q, Van Eerd D, Irvin E. Searching for grey literature for systematic reviews: challenges and benefits. Research Synthesis Methods. 2014;5(3):221-34.

40. Tricco AC. Doing a scoping review: what they are and how you can do them [Internet]. 2017 [cited 23 Feb 2021]. Available from: https://training.cochrane.org/resource/scoping-reviews-what-they-areand-how-you-can-do-them.

41. Benzies KM, Premji S, Hayden KA, Serrett K. State-of-the-evidence reviews: advantages and challenges of including grey literature. Worldviews on Evidence-Based Nursing. 2006;3(2):55-61. 
42. Palladino DK, Helgeson VS, Reynolds KA, Becker DJ, Siminerio LM, Escobar O. Emerging adults with type 1 diabetes: a comparison to peers without diabetes. Journal of Pediatric Psychology. 2013;38(5):506-17.

43. Tricco AC, Lillie E, Zarin W, O'Brien KK, Colquhoun H, Levac D, et al. PRISMA extension for scoping reviews (PRISMA-ScR): checklist and explanation. Annals of Internal Medicine. 2018;169(7):467-73.

44. Qualter P, Rouncefield-Swales A, Bray L, Blake L, Allen S, Probert C, et al. Depression, anxiety, and loneliness among adolescents and young adults with IBD in the UK: the role of disease severity, age of onset, and embarrassment of the condition. Quality of Life Research. 2020:1-10.

\section{Supplementary Files}

This is a list of supplementary files associated with this preprint. Click to download.

- AdditionalFile1ExamplesearchstrategyforPsyclnfo.docx

- AddtionalFile2CompletedPRISMAPChecklist.docx 